\title{
VALIDADE DA MEDIDA DO CONSUMO MÁXIMO DE OXIGÉNIO PREDITO PELO TESTE DE COOPER DE 12 MINUTOS EM ADULTOS JOVENS SEDENTÁRIOS
}

\author{
AUTORES \\ Eduardo Caldas Costa ${ }^{1}$ \\ VALIDADE DA MEDIDA \\ DO CONSUMO MÁXIMO \\ DE OXIGÉNIO PREDITO \\ PELO TESTE DE COOPER \\ DE 12 MINUTOS EM ADULTOS \\ JOVENS SEDENTÁRIOS \\ 4(3): 5-10
}

\section{PALAVRAS-CHAVE}

resistência cardiorespiratória; idosas; sobrepeso; atividade física.

\section{KEYWORDS}

cardiorespiratory resistance; elderly; overweight; physical activity.

\section{RESUMO}

O objetivo do estudo foi investigar se um programa de atividade física promove melhoras significativas na capacidade cardiorespiratória de idosas. Participaram do estudo 71 gerontes do sexo feminino, sendo divididas em quatro grupos nas respectivas faixas etárias: $\mathbf{G 1}$ [60-64 anos; $n=29$ ), $G 2$ (65-69 anos; $n=22$ ), G3 (70-74 anos; $n=11$ ) e G4 (75-79 anos; $n=09$ ). A aptidão cardiorespiratória foi avaliada através do teste de caminhada de 6 minutos de Rikli e Jones (1998). Foi utilizado o teste Shapiro-Wilk, para verificação da gaussianidade da amostra, com nível de significância $<0,05$, utilizou-se 0 programa SPSS 10.0 for Windows.
Analisando a variável distância percorrida, obteve-se as seguintes médias:

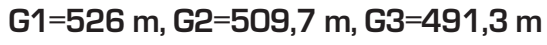
e $\mathrm{G} 4=479,4 \mathrm{~m}$, pode-se concluir que somente o G1 não obteve seu valor dentro da faixa de referência. Considerando a possibilidade da não especificidade da prescrição do exercício físico para cada faixa etária apresentada pelos grupos estudados, o programa de atividade física realizado foi capaz de proporcionar a 60,5\% daquelas idosas, um perfil satisfatório para a resistência cardio-respiratória. 


\section{INTRODUC̣ÃO}

0 primeiro trabalho envolvendo a realização de um teste para análise da capacidade funcional foi publicado por Master e Oppenheimer em 1929, utilizando uma escada de dois degraus com o objetivo de avaliar a capacidade do coração ao exercício por meio de respostas da freqüência cardíaca $(F C)$ e da pressão arterial (PA). A esse trabalho é também creditada a primeira descrição de um protocolo para teste de esforço ${ }^{6}$.

As bases da moderna eletrocardiografia de esforço foram estabelecidas nos anos 50. Wood na referida década enfatizou a necessidade de que fosse exercitada a capacidade máxima de cada indivíduo, resultando em FC acima de $90 \%$ da prevista. Seu protocolo consistiu na subida de 84 degraus de uma escadaria próxima ao hospital em que trabalhava, com o registro imediato do eletrocardiograma. Em 1954, Astrand e Rhyming estabeleceram as bases fisiológicas para o método, correlacionando o consumo de oxigênio $\left[\mathrm{VO}_{2}\right]$ com a $\mathrm{FC}$ em níveis submáximos de exercício ${ }^{23}$.

O consumo máximo de oxigênio [ $\mathrm{VO}_{2}$ máx] pode ser definido como a máxima capacidade de captação (pulmões), transporte (coração e vasos) e utilização do oxigênio (principalmente pelos músculos] durante exercício dinâmico envolvendo grande massa muscular corporal ${ }^{3}$. Apesar do consumo máximo de oxigênio $\left[\mathrm{VO}_{2} \mathrm{máx}\right]$ ser um índice objetivo do grau de esforço realizado, assim como de funcionalidade, ele só foi utilizado nos protocolos de teste de esforço a partir de 1955 , quando as técnicas de medida de gases tornaram-se disponíveis. 0 ano de 1956 pode ser considerado um marco na evolução da ergome- tria, pela introdução da esteira rolante e o respectivo protocolo para utilização deste novo equipamento, inserido por Robert Bruce ${ }^{23}$.

Em 1968, Kenneth Cooper ${ }^{8}$ ao realizar testes de campo com militares da Força Aérea Americana descreveu um procedimento avaliativo (teste de 12 minutos) para a estimativa do $\mathrm{VO}_{2}$ máx. Este consistiu em uma modificação do teste de corrida de 15 minutos desenvolvido anteriormente por Balke, também com militares ${ }^{7}$.

$0 \mathrm{VO}_{2}$ máx é um parâmetro usado para a avaliação da função cardiorrespiratória máxima e reserva funcional $^{12}$. Além disso, a mensuração do $\mathrm{VO}_{2}$ máx pode ser indicada por várias razões, desde a análise da função cardiorrespiratória, em pneumo e cardiopatas, até a predição de desempenho em atletas ${ }^{20}$. 0 $\mathrm{VO}_{2}$ máx também serve como um indicador independente de mortalidade por todas as causas, e principalmente as de etiologia cardiovascular $^{5,10,14,15,16}$.

Atualmente, os testes de aptidão física apresentam alguns objetivos substanciais, dentre os quais estão: fornecimento de dados úteis no desenvolvimento da prescrição de exercício, coleta de dados que permitam a avaliação do progresso dos participantes em programa de exercício, motivação dos indivíduos, estabelecendo objetivos de aptidão física alcançáveis, orientação aos participantes sobre os conceitos de aptidão física, estado de aptidão individual, além de estratificação de risco? .

O critério tradicional de avaliação da capacidade cardiorrespiratória (método direto) envolve análises de amostras de ar expirado coletadas enquanto a pessoa realiza exercício em intensidade progressiva. Essa medida direta do $\mathrm{VO}_{2}$ máx é feita submetendo o indivíduo a um teste ergométrico com cargas crescentes e analisando as frações expiradas de oxigênio e dióxido de carbono durante o esforço, além da ventilação pulmonar ${ }^{2}$. Esse tipo de procedimento é considerado padrão-ouro para esse fim ${ }^{9}$.

Entretanto, seu custo é alto, são necessários equipamentos sofisticados, mão-de-obra especializada para a administração do teste, maior quantidade de tempo com cada avaliado e ainda maior motivação do indivíduo, pois geralmente é realizada em ambiente de laboratório ${ }^{2}$. Contudo, o $\mathrm{VO}_{2}$ máx também pode ser medido a partir da intensidade do esforço máxima usando equações de previsão (método indireto). Esse tipo de estimativa é considerado o próximo método mais exato de mensuração do consumo máximo de oxigênio. Como a medida direta do $\mathrm{VO}_{2}$ máx geralmente não é possível, muitos procedimentos para calculá-lo têm sido desenvolvidos. Novas propostas avaliativas vêm sendo validadas, a partir da análise de correlação entre $\mathrm{VO}_{2}$ máx medido diretamente e determinado teste de desempenho físico ${ }^{2}$.

Os testes de campo, nos quais o cálculo do $\mathrm{VO}_{2}$ máx é feito através de equações baseadas em tempo ou distância pré-estabelecidos, são exemplos desse tipo de correlação. Nesse caso, podem ser avaliadas várias pessoas ao mesmo tempo com baixo custo e fácil aplicabilidade $^{2}$. Sua validade, entretanto, muitas vezes é questionada, tendo em vista que muitas dessas equações são específicas para grupos populacionais pré-determinados ${ }^{4}$.

Um dos testes de campo mais utilizados para avaliação do $\mathrm{VO}_{2}$ máx é o teste de Cooper de 12 minutos. Uma de suas vantagens é a facilidade de administração. Por outro 
lado, apresenta algumas limitações substanciais, como nível individual de motivação e capacidade de desenvolvimento de ritmo adequado no decorrer do teste ${ }^{2}$.

Desta forma, toda vez que, por falta de disponibilidade, seja financeira, estrutural ou tecnológica, venha a ser realizada uma avaliação mais "simples" (como, por exemplo, os testes de predição de $\mathrm{VO}_{2}$ máx], deve-se conhecer, ao menos, limitacões e possibilidades de erro envolvidas na sua execução e coleta de dados, quando comparadas aos exames com medida direta de gases ${ }^{20}$. Com o exposto, torna-se relevante o conhecimento da validade de procedimentos avaliativos de baixo custo que inferem a capacidade funcional dos indivíduos através da predição do consumo máximo de oxigênio. Portanto, o propósito do presente estudo foi analisar a validade da medida do $\mathrm{VO}_{2}$ máx predito pelo teste de Cooper de 12 minutos em adultos jovens sedentários.

\section{METODOLOGIA}

\section{Amostra}

A população envolvida no presente estudo foi de 45 alunos matriculados no 9 e 10 período do curso de Fisioterapia da Universidade Potiguar (UNP). Os critérios de seleção da amostra foram: ser do sexo masculino, apresentar faixa etária entre 20 e 29 anos, não apresentar disfunção óssea, muscular, articular e cardiovascular e não estar inserido em nenhum programa de exercício físico regular. Fizeram parte do estudo 11 voluntários com idade média de $24 \pm 1,8$ anos, massa corporal de 78,44 $\pm 10,82 \mathrm{~kg}$, estatura de $174 \pm 8,2 \mathrm{~cm}$ e IMC de $26 \pm 3,8 \mathrm{~kg} / \mathrm{m}^{2}$.

\section{Procedimentos}

Esta pesquisa foi aprovada no Comitê de Ética da UNP em 28/11/ /2006, processo nํ 129/2006.

Primeiramente foi realizado um levantamento estatístico através da direção do curso de Fisioterapia da UNP, sobre os alunos do sexo masculino com faixa etária entre 20-29 anos matriculados no 9응 e 10 período do referido curso. Essas pessoas foram contactadas e esclarecidas sobre os objetivos da pesquisa. Os voluntários que aceitaram participar do estudo assinaram um termo de consentimento livre e esclarecido.

Após a definição da amostra, informações pré-teste de acordo com recomendações do American College of Sports Medicine ${ }^{2}$ foram proferidas aos participantes (não fazer atividade física extenuante 24 horas antes dos testes; não ingerir café, bebida alcoólica nem fumar três horas antes dos testes; usar roupas leves nas avaliações e dormir adequadamente 6 a 8 horas no dia anterior dos testes], sendo agendada a data da ergoespirometria e do teste de Cooper de todos os indivíduos. As avaliações ocorreram com um intervalo de no mínimo 48 horas e no máximo de 72 horas entre uma e outra.

Os indivíduos foram submetidos a teste ergométrico em esteira rolante seguindo um protocolo de rampa com intensidade progressiva adaptado de Tebexreni et al. ${ }^{23}$. Inicialmente foi realizado um breve aquecimento (3 minutos com velocidade de 2,5 km/h sem inclinação]. Após esse período, o teste foi iniciado com velocidade de $3 \mathrm{~km} / \mathrm{h}$ sem inclinação, onde a partir de então, houve incremento de carga (velocidade e inclinação) a cada 6 segundos até que os indivíduos atingissem a exaustão entre 8 e 12 minutos $^{17,19,25,11}$. Durante o teste o ar expirado foi coletado pelo analisador de gases metabólicos. A recuperação teve início imediatamente após os indivíduos atingirem o pico de esforço, sendo realizada no primeiro minuto a uma velocidade de $50 \%$ da final atingida no teste (sem inclinação) e no segundo minuto a $25 \%$.

Utilizou-se para o estabelecimento do consumo máximo de oxigênio os seguintes dados: a) presença de QR $\left[\mathrm{VCO}_{2} / \mathrm{VO}_{2}\right]>1.1$; b] existência de um limiar anaeróbico (LA); c) ventilação pulmonar [VE] > 60\% da máxima prevista, e; d) eventual presença de um platô no $\mathrm{VO}_{2}$ diante de um aumento na carga de esforco. Estes dados, concomitante à avaliação da freqüência cardíaca máxima (FCM) atingida e a sensação subjetiva de esforço, foram usados também para assegurar um teste máximo ${ }^{25}$.

0 teste de Cooper de 12 minutos foi realizado na pista de atletismo da Universidade Federal do Rio Grande do Norte [UFRN]. Os indivíduos correram e/ou andaram ao redor da mesma a máxima distância possível no tempo pré-estabelecido. Cones de sinalização foram colocados a cada $50 \mathrm{~m}$ para que fosse facilitada a mensuração (através de trena) da distância máxima alcançada ao final do teste. Com os valores de metragem individualmente percorridos foi calculado o $\mathrm{VO}_{2}$ máx predito para cada participante, através da fórmula proposta por Cooper ${ }^{4}$ :

$\left[\mathrm{VO}_{2} \mathrm{MÁX}=(\mathrm{m}-504,9) / 44,73\right]$ onde " $m$ " indica a distância em metros percorrida pelo indivíduo.

\section{Estatística}

Os dados foram armazenados no programa Excel e analisados no pacote estatístico SPSS $\AA$, versão 


\begin{tabular}{lcc}
\hline Variáveis & Média & $\begin{array}{c}\text { Desvio- } \\
\text {-padrão }\end{array}$ \\
\hline Idades (anos) & 24 & 1,8 \\
Massa corporal & 78,4 & 11,2 \\
Estatura (cm) & 174 & 8,2 \\
IMC & 26 & 3,8 \\
PAS (mmHg) & 122 & 10,1 \\
PAD (mmHg) & 71 & 5,5 \\
FCrep (bpm) & 73 & 10,1 \\
\hline
\end{tabular}

TABELA1

Caracterização da amostra $(n=11)$.

\begin{tabular}{lcc} 
Variáveis & Média & $\begin{array}{c}\text { Desvio- } \\
\text {-padrăo }\end{array}$ \\
\hline Distância $(\mathrm{m})$ & 2160 & 232,9 \\
Tempo final $(\mathrm{min})$ & 10 & 0,8 \\
\hline & Fonte: dados da pesquisa
\end{tabular}

\section{TABELA2}

Distância percorrida no teste de Cooper de 12 minutos e tempo final da ergoespirometria $(n=11)$.

14.0 para Windows. Para caracterização da amostra e disposição dos resultados foi usada a estatística descritiva (média e desvio-padrão]. Para análise de diferença entre o $\mathrm{VO}_{2}$ máx medido através da ergoespirometria e do $\mathrm{VO}_{2}$ máx predito pelo teste de Cooper de 12 minutos foi utilizado o teste $U$ de Mann-Whitney acatando-se um nível de significância de 5\%.

\section{RESULTADOS E DISCUSSÃO}

A tabela 1 mostra as medidas descritivas da amostra do estudo no que se refere às variáveis: idade, massa corporal, estatura, índice de massa corpórea (IMC), pressão arterial sistólica (PAS), pressão arterial diastólica (PAD) e freqüência cardíaca de repouso (FCrep).
De acordo com Cooper ${ }^{4}$ a distância média alcançada pelos indivíduos equivale a uma classificação regular de aptidão aeróbia. Já o tempo médio de 10 minutos atingido pelos sujeitos na ergoespirometria se situou na faixa considerada ideal para esse tipo de procedimento, podendo essa zona ser estendida entre 8 e 12 minutos ${ }^{17,17,25,11}$. As médias de distância percorrida no teste de 12 minutos e o tempo do teste na ergoespirometria do presente estudo são apresentadas na tabela 2 .

A tabela 3 exibe os resultados da medida do consumo máximo de oxigênio: medida direta e indireta. Conforme os resultados mostrados a seguir, o valor médio do $\mathrm{VO}_{2}$ máx obtido através da ergoespirometria foi maior do que o predito pelo teste de Cooper de 12 minutos. Além disso, foi constatada maior variabilidade nos valores de $\mathrm{VO}_{2}$ máx preditos pelo teste de Cooper, evidenciada a partir da observação do desvio-padrão presente na tabela que se segue.

Grant et al. ${ }^{13}$ observaram, através do teste de Cooper e ergoespirometria, valores médios de $\mathrm{VO}_{2}$ máx de $60,6 \mathrm{ml} / \mathrm{kg} / \mathrm{min}$ e $60,1 \mathrm{ml} / \mathrm{kg} / \mathrm{min}$, respectivamente. Esses valores bem superiores aos encontrados no presente estudo podem ser justificados pela diferente amostra que constituiu o trabalho de Grant e colaboradores, sendo essa formada por jovens universitários praticantes de esporte com freqüência regular. Dados semelhantes aos encontrados no presente estudo em relação ao consumo máximo de oxigênio (medido através da ergoespirometria) foram encontrados por Rondon et al. $^{18}$, onde ao analisarem jovens do sexo masculino, saudáveis, com idade média de 29 anos verificaram o valor médio do $\mathrm{VO}_{2}$ máx de 42,1 $\mathrm{ml} / \mathrm{kg} / \mathrm{min}$.
Numa metanálise sobre envelhecimento, declínio da capacidade aeróbia máxima e sua relação com nível de treinamento, foi observada a média do $\mathrm{VO}_{2}$ máx no grupo sedentário de $36,9 \mathrm{ml} / \mathrm{kg} / \mathrm{min}$ com desvio-padrão de $8,2^{24}$. Esse elevado desvio-padrão provavelmente deveu-se ao envolvimento de grupos etários distintos, variando de 20 a mais de 70 anos. Entretanto, harmonizando os resultados encontrados pelos autores com a faixa etária considerada no presente trabalho [20-29 anos] os valores de $\mathrm{VO}_{2}$ máx encontram-se similares, ou seja, próximos à faixa de $40 \mathrm{ml} /$ $/ \mathrm{kg} / \mathrm{min}$.

Num levantamento do número de exames ergoespirométricos efetivados no CEMAFE (Centro de Medicina da Atividade Física e do Esporte) da Escola Paulista de Medicina (UNIFESP), constatou-se que 203 indivíduos sedentários submeteram-se a tal teste para avaliação da capacidade aeróbia e determinação dos índices de limitação funcional $^{23}$. $\mathrm{O}$ valor médio de $\mathrm{VO}_{2}$ máx encontrado foi de $36,17 \mathrm{ml} / \mathrm{kg} / \mathrm{min}$ com desvio-padrão de 6,52. Contudo, a média de idade dos indivíduos avaliados foi de 40,53 anos com desvio-padrão de 12,56, o que pode explicar a alta variabilidade encontrada para a variável $\mathrm{VO}_{2}$ máx e permite justificar a ocorrência de um menor valor médio da mesma para essa população.

Cooper8 ao avaliar 115 indivíduos da Força Aérea Americana verificou uma alta correlação entre o consumo máximo de oxigênio estabelecido pelo seu teste de 12 minutos e o respectivo $\mathrm{VO}_{2}$ máx obtido em laboratório $(r=0,90)$. A idade média dos indivíduos foi de 22 anos com variação entre 17 e 54 anos. Os valores de $\mathrm{VO}_{2}$ máx alcançados pelos indivíduos variaram de 31 a $59 \mathrm{ml} / \mathrm{kg} / \mathrm{min}$. 
Numa comparação entre vários métodos de predição de consumo máximo de oxigênio, incluindo teste de Cooper de 12 minutos, shuttle run de multiestágios progressivos e teste de bicicleta submáximo, em adultos jovens do sexo masculino, praticantes de esporte, foi verificado que o teste de Cooper obteve maior correlação com a medida direta do $\mathrm{VO}_{2}$ máx $(r=0,92)^{13}$, dado semelhante ao encontrado por Cooper $^{8}$, tendo os outros testes um viés de subestimação dos reais valores encontrados em laboratório. MacArdle et al. ${ }^{21}$ destacam um problema em potencial ao se comparar testes preditivos para avaliação de consumo máximo de oxigênio com avaliação por medida direta em grupos homogêneos. Os mesmos constataram que quando o teste de Cooper original foi restringido para avaliação de jovens universitários do sexo masculino a correlação caiu de 0,90 para 0,59. McCutcheon et al. ${ }^{22}$ encontraram uma alta correlação entre o consumo máximo de oxigênio predito pelo teste de Cooper de 12 minutos e a medida direta dessa variável [ $\mathrm{VO}_{2} \mathrm{máx}$ em adultos jovens do sexo masculino e feminino, com idade média de 25 anos, sendo o referido $r=0,84$. Entretanto, apesar da alta correlação verificada, os autores observaram uma subestimação média dos valores de $\mathrm{VO}_{2}$ máx preditos pelo teste de Cooper de 4 $\mathrm{ml} / \mathrm{kg} / \mathrm{min}$ em relação aos testes laboratoriais. Esse achado se assemelha à média de subestimação encontrada no presente estudo, $6 \mathrm{ml} / \mathrm{kg} / \mathrm{min}$.

Quando se avalia um grupo com grande variação de idade, alta correlação estatística é esperada'.

Além disso, resultados muito heterogêneos de uma variável estudada

\begin{tabular}{|c|c|c|c|c|}
\hline Variáveis & Média & Desvio-padrão & Diferença [\%] & p-valor \\
\hline $\mathrm{VO}_{2}$ máx direto $(\mathrm{ml} / \mathrm{kg} / \mathrm{min})$ & 43,18 & 3,7 & \multirow{2}{*}{$14,7 \%$} & \multirow{2}{*}{0,005 * } \\
\hline $\mathrm{VO}_{2}$ máx indireto $(\mathrm{ml} / \mathrm{kg} / \mathrm{min})$ & 36,94 & 5,2 & & \\
\hline \multicolumn{5}{|l|}{ Fonte: dados da pesquisa } \\
\hline & & & & TABELA3 \\
\hline \multicolumn{5}{|c|}{ Caracterização da amostra ( $n=11)$. } \\
\hline
\end{tabular}

também favorecem uma correlação estatística elevada. No estudo original de Cooper ${ }^{8}$ a faixa estaria dos indivíduos estudados variou de 17 a 54 anos e os resultados de $\mathrm{VO}_{2}$ máx de 31 a $59 \mathrm{ml} / \mathrm{kg} / \mathrm{min}$. Logo, esses aspectos devem ser considerados quando esse teste é aplicado em determinada população.

\section{CONCLUSÃO}

De acordo com os dados apresentados, é possível concluir que houve diferença estatisticamente significante $(p<0,05)$ entre o $\mathrm{VO}_{2}$ máx predito pelo teste de Cooper de 12 minutos em comparação ao obtido na ergoespirometria. Em média, o valor predito pelo teste de Cooper de 12 minutos foi $14,7 \%$ menor em relação à ergoespirometria. Desta forma, fica perceptivel que para a população estudada o teste de Cooper de 12 minutos não apresentou boa validade na predição do consumo máximo de oxigênio, subestimando, portanto, os reais valores obtidos através do teste padrão-ouro (ergoespirometria).

Com isso, possíveis informações geradas em relação ao estado de aptidão física e capacidade funcional dos indivíduos estudados, a partir dos resultados preditos pelo teste de Cooper de 12 minutos, podem ser consideradas aquém da real situação, fato notório a partir do viés de subestimação apresentado pelo referido teste no presente trabalho.

0 número pequeno da amostra impede que afirmações mais concretas em relação à validade do teste de Cooper de 12 minutos para os fins estudados sejam realizadas. Logo, se faz necessário outros trabalhos semelhantes, e com amostras mais representativas em termos quantitativos para esclarecer tais aspectos. Parece também ser importante estudos envolvendo outras populações: gênero feminino, faixas etárias distintas e diferentes níveis de aptidão física, o que permitiria um melhor entendimento quanto aos resultados obtidos no presente trabalho e sua aplicação em diversas populações.

\section{CORRESPONDÊNCIA}

Eduardo Caldas Costa

Av. Rui Barbosa 1100.

Bairro: Lagoa Nova Natal-RN.

CEP: 59056300

Contacto: (84) 32343640

(84) 99256000

E-mail:

eduardocaldascosta@hotmail.com 


\section{REFERÊNCIAS}

1. Anderson GS (1992). A comparison of predictive tests of aerobic capacity. Can J Sport Sci 17:304-308.

2. American College of Sports Medicine (2000). ACSM's Guidelines for Exercise Testing and Prescription. $6^{\underline{a}}$ ed. Baltimore: Lippincott Williams and Wilkins.

3. Astrand PO; Rodahl K (1986). Textbook of work physiology. New York: McGraw-Hill.

4. Basset DR; Howley ET (2000). Limiting factors for maximum oxygen uptake and determinants of endurance performance. Med Sci Sports Exerc 32:70-84.

5. Blair SN; Kohl HW; Barlow CE; Paffenbarger Jr RS; Gibbons LW; Macera CA (1995). Changes in physical fitness and all-cause mortality. A prospective study of healthy and unhealthy men. JAMA 273:1093$-1098$.

6. Chalela WA; Moffa PJ (2005). Teste Ergométrico. In: Negrão CE, Barreto ACP [Eds]. Cardiologia do exercício: do atleta ao cardiopata. São Paulo: Manole, 92-127.

7. Cooper CB; Storer TW (2005). Teste ergométrico: aplicações práticas e interpretação. Rio de Janeiro: Revinter.

8. Cooper KH (1968). A means of assessing maximal oxygen intake. Correlation between field and treadmill testing. JAMA 203:201-204.

9. Diaz FJ; Montano JG; Melchor MT; Guerrero JH; Tovar JA (2000). Validation and reliability of the 1,000 meter aerobic test. Rev Invest Clin 52:44-51.

10. Erikssen G; Liestol K; Bjornholt J; Thaulow E; Sandvik L; Erikssen J
(1998). Changes in physical fitness and changes in mortality. Lancet 352:759-762.

11. Fletcher GF; Balady CG; Froelicher VF; Amsterdan EA; Chaitman B; Eckel R; Fleg J; Froelicher VF; Leon AS; Pina IL; Rodney R; Simons-Morton DG; Williams MA; Bazarre $T$ (2001). Exercise standards for testing and training. A statement for healthcare professionals from the American Heart Association. Circulation 104:1694-740.

12. Granja Filho PCN; Pompeu FAMS; Souza e Silva APR (2005). A validade da determinação do $\mathrm{VO}_{2}$ máx e do limiar anaeróbio. Rev Bras Med Esporte 11:167-171.

13. Grant S; Corbett K; Amjad AM; Wilson J; Aitchison T (1995). A comparison of methods of predicting maximum oxygen uptake. $\mathrm{Br} J$ Sports Med 29:147-152.

14. Kavanagh T; Mertens DJ; Hamn LF; Beyene J; Kennedy J; Corey P; Shepard RJ (2002). Prediction of Long-Term Prognosis in 12.169 Men Referred for Cardiac Rehabilitation. Circulation 106:666-671.

15. Lamonte $\mathrm{MJ}$; Eisenman PA; Adams TD; Shultz BB; Ainsworth BE; Yanowitz FG (2000). Cardiorespiratory Fitness and Coronary Heart Disease Risk Factors: The LDS Hospital Fitness Institute Cohort. Circulation 102:1623-1628.

16. Laukkanen JA; Lakkata TA; Rauramaa R; Kuhanen R; Venalainen JM; Salonen R; Salonen JT [2001). Cardiovascular fitness as a predictor of mortality in men. Arch Intern Med 161:825-831.

17. Myers J; Buchanan N; Walsh D; Kraemer M; McAuley P; Hamilton-Wessler M; Froelicher VF (1991).
Comparison of the ramp versus standard exercise protocols. JACC 17:1334-1342.

18. Rondon MUPB; Forjaz CLM; Nunes N; Amaral SL; Barreto ACP; Negrão CE (1998). Comparação entre a prescrição de intensidade de treinamento físico baseada na avaliação ergométrica convencional e na ergoespirométrica. Arq Bras Cardiol 70:159-166.

19. Serra S. Considerações sobre ergoespirometria (1997). Arq Bras Cardiol 68:301-304.

20. Silva AC; Torres FC (2002). Ergoespirometria em atletas paraolímpicos brasileiros. Rev Bras Med Esporte 8:107-116.

21. McArdle WD; Katch Fl; Katch VL [1986). Exercise physiology, energy, nutrition and human performance. $2^{a}$ ed. Philadelphia: Lea and Febiger. 22. McCutcheon MC; Sticha SA; Giese MD; Nagle FJ (1990). A further analysis of the twelve minute run prediction of maximal aerobic power. Res Q Exerc Sport 61:280$-283$.

23. Tebexreni AS; Lima EV; Tambeiro VL; Barros Neto TL (2001). Protocolos tradicionais em ergometria, suas aplicações práticas versus protocolo de rampa. Rev Soc Cardiol Estado de São Paulo 11:519-528.

24. Wilson TM; Tanaka W (2000). Meta-analysis of the age-associated decline in maximal aerobic capacity in men: relation to training status. Am $\checkmark$ Physiol Heart Circ Physiol 278: :829-834.

25. Yazbek P; Carvalho RT; Sabbag LMS; Battistella LR (1998). Ergoespirometria. Teste de Esforço Cardiopulmonar, Metodologia e Interpretação. Arq Bras Cardiol 71:719-724. 